\title{
Axial spondyloarthritis in the USA: diagnostic challenges and missed opportunities-comments on the article by Danve A \& Deodhar A
}

\author{
Sasan Fallahi ${ }^{1,2}$ \\ Received: 6 January 2019 / Accepted: 15 January 2019 / Published online: 24 January 2019 \\ (C) International League of Associations for Rheumatology (ILAR) 2019
}

To editor:

I read the interesting manuscript entitled Axial spondyloarthritis in the USA: diagnostic challenges and missed opportunities which has been recently published by Danve A \& Deodhar A in Clinical Rheumatology [1]. They included in their paper: "none of these physical examination techniques, including spine mobility measurements, reliably distinguish inflammatory diseases of the spine, such as axial spondyloarthritis, from common degenerative diseases of the spine". I am opposed to this opinion. Due to lack of reliable diagnostic biomarkers, an accurate physical examination along with patient history is the best way to diagnose on time. As Danve A \& Deodhar A believe, the low awareness of non-rheumatology physician about spondyloarthropathy is one of the causes of delayed diagnosis in this disease [1]. However, accidental coincidence of lumbar discopathy as a common cause of back pain and as a result, the lack of attention to inflammatory manifestations can be one of the other causes of diagnostic delay in these patients [2]. The gradual and insidious progress of disease in some patients which may lead to the patient's compliance with their illness and consequently, lack of timely referral to the doctor, may be another reason for this delay. Negative HLA-B27 (about 10\% of patients with ankylosing spondylitis (AS) in the USA and Europe and $30 \%$ in Iran and other Middle Eastern countries), low education level of patients, and the enthesitis have been reported as other associated factors of diagnostic delay in patients with AS in Fallahi et al. study [3]. Also, negative family history of HLA-B27, absence of extra-articular

Sasan Fallahi

sfallahi@sina.tums.ac.ir; drsfallahi@gmail.com

1 Internal Medicine Division, Baharloo Hospital, Tehran University of Medical Sciences, Behdari Street, South Kargar Street, Tehran, Iran

2 Rheumatology Research Center, Tehran University of Medical Sciences, Tehran, Iran manifestations, and juvenile age at onset were among other factors associated with diagnostic delay in some studies [4-6]. In conclusion, spending adequate time for taking history and physical examination especially in patients with low education levels, raising awareness of other physicians involved with back pain, timely referral of patients to the rheumatologist, attention to the presence of enthesitis including heel pain, and finally refrain from relying too much on laboratory markers include HLA-B27, erythrocyte sedimentation rate, and C-reactive protein may help reduce diagnosis delay in patients with AS.

\section{Compliance with ethical standards}

Disclosure None.

Publisher's note Springer Nature remains neutral with regard to jurisdictional claims in published maps and institutional affiliations.

\section{References}

1. Danve A, Deodhar A (2018) Axial spondyloarthritis in the USA: diagnostic challenges and missed opportunities. Clin Rheumatol. https://doi.org/10.1007/s10067-018-4397-3

2. Gerdan V, Akar S, Solmaz D, Pehlivan Y, Onat AM, Kisacik B, Sayarlioglu M, Erhan C, Tezcan ME, Ozturk MA, Onen F, Akkoc $\mathrm{N}$ (2012) Initial diagnosis of lumbar disc herniation is associated with a delay in diagnosis of ankylosing spondylitis. J Rheumatol 39:1996-1999

3. Fallahi S, Jamshidi AR (2015) Diagnostic delay in ankylosing spondylitis: related factors and prognostic outcomes. Arch Rheumatol 31: 24-30

4. Dincer U, Cakar E, Kiralp MZ, Dursun H (2008) Diagnosis delay in patients with ankylosing spondylitis: possible reasons and proposals for new diagnostic criteria. Clin Rheumatol 27:457-462

5. Aggarwal R, Malaviya AN (2009) Diagnosis delay in patients with ankylosing spondylitis: factors and outcomes-an Indian perspective. Clin Rheumatol 28:327-331

6. Feldtkeller E, Khan MA, van der Heijde D, van der Linden S, Braun J (2003) Age at disease onset and diagnosis delay in HLAB27 negative vs. positive patients with ankylosing spondylitis. Rheumatol Int 23:61-66 